\title{
Compte rendu de Art de l'archipel Bismarck, de Kevin Conru (éd.)
}

\section{Gilles Bounoure}

\section{OpenEdition}

1 Journals

\section{Édition électronique}

URL : http://journals.openedition.org/jso/7178

DOI : $10.4000 /$ jso. 7178

ISSN : $1760-7256$

\section{Éditeur}

Société des océanistes

\section{Édition imprimée}

Date de publication : 15 décembre 2014

Pagination : 269-272

ISBN : 978-2-85430-118-2

ISSN : 0300-953x

\section{Référence électronique}

Gilles Bounoure, "Compte rendu de Art de l'archipel Bismarck, de Kevin Conru (éd.) », Journal de la Société des Océanistes [En ligne], 138-139 | 2014, mis en ligne le 15 décembre 2014, consulté le 23 septembre 2020. URL : http://journals.openedition.org/jso/7178; DOI : https://doi.org/10.4000/jso 7178

Ce document a été généré automatiquement le 23 septembre 2020.

(c) Tous droits réservés 


\title{
Compte rendu de Art de l'archipel Bismarck, de Kevin Conru (éd.)
}

\author{
Gilles Bounoure
}

\section{RÉFÉRENCE}

CONRU Kevin (éd.), 2013. Art de l'archipel Bismarck, textes de Klaus-Jochen Krüger, Bart

Van Bussel, K. Conru, Ingrid Heermann, photographies d'Hughes Dubois, Milan, éditions 5 Continents, 328 p., bibliogr., carte, très nombreuses illustrations noir et blanc et couleur.

1 Les proportions de ce volume sont excentriques sinon tout à fait inédites (362 x 280 x 34 $\mathrm{mm}, 3,4 \mathrm{~kg}$ environ). Il faut remonter assez loin dans l'histoire de l'édition pour trouver des exemples approchants dans le domaine des arts océaniens, les albums de Meyer et Parkinson édités par le musée de Dresde (1890 et 1895), ceux de Portier et Poncetton (1930, 1931), ou encore la sélection de photos préfacée par Carl A. Schmitz (1962) et celle de Jean Guiart (1965), de formats légèrement supérieurs, moins épais et plus maniables, mais tous impossibles à ranger dans des bibliothèques à rayonnages standard. Faire exception au titre d'objets eux-mêmes exceptionnels, reproduits avec une qualité et des dimensions également hors du commun, telle semble l'ambition à l'origine de tous ces ouvrages, et du présent volume en particulier.

2 Voyageur, collectionneur membre de la Pacific Arts Association, K. Conru est avant tout marchand d'art. À côté de catalogues prolongeant ses vitrines, il a suscité des publications moins directement commerciales, notamment trois volumes exploitant un fonds de photographies ethnographiques acquis de la famille d'Hugo Adolf Bernatzik, et plus récemment un «beau livre » consacré aux arts des îles Salomon (2008), naguère décrit dans cette rubrique (Jso 130-131). Il s'était alors attaché la participation de Deborah Waite, spécialiste renommée de ces arts, rôle dévolu dans le présent ouvrage à I. Heermann, autre autorité reconnue pour ses publications, ses expositions et 
l'ensemble de son travail à la tête du département océanien du Linden Museum de Stuttgart depuis plusieurs décennies (par exemple 2001, 2009).

3 Contrairement au volume précédent limité à la seule « collection Conru » d'objets des îles Salomon, l'éditeur a fait appel ici à d'autres collectionneurs d'Europe, d'Amérique du Nord et d'Australie - pour autant qu'on puisse identifier les possesseurs actuels des pièces reproduites, qui ne sont pas tous cités dans les légendes pourtant disertes sur le parcours de ces pièces ${ }^{1}$, ni dans le reste de l'ouvrage. Une proportion infime (six objets, soit environ $3 \%$ de l'ensemble) venant d'institutions muséales, les lecteurs potentiels se voient proposer le privilège d'approcher de trésors demeurés en mains privées, voire encore inédits. On pourra toujours sourire de cette vieille recette éditoriale, elle n'en est pas moins inusable et doublement efficace, suscitant la curiosité et la satisfaisant assez souvent, jusqu'à procurer parfois des découvertes d'ampleur variée.

Inédits, les objets rassemblés dans Art de l'archipel Bismarck sont loin de l'être tous. Nombre d'entre eux avaient été présentés lors d'une exposition bruxelloise fondée sur le même principe d'emprunts à de grandes collections privées européennes, pour figurer au catalogue édité à cette occasion (Herreman 2008, décrit dans le JSO 128). Pour ces pièces, les légendes se sont seulement étoffées, d'une publication à l'autre, de « pedigrees » retraçant tout ou partie de leur circulation une fois parvenus en Occident et suggérant l'ancienneté de leur collecte, sinon de leur fabrication, mais sans les notices dont les accompagnait le catalogue de 2008 et dont la substance est à chercher dans le texte courant. On n'observe malheureusement aucune avancée dans la description même des objets et des matières mises en œuvre, uniformément et paresseusement qualifiées de "pigments », "coquillages ", "bois », etc., malgré des différences d'aspect et d'usage largement connues des spécialistes.

5 Le volume s'ouvre sur un long exposé historique de Kl.-J. Krüger, "Colons, administrateurs, chercheurs et explorateurs. Les campagnes de collecte dans l'archipel Bismarck» (pp. 11-41). L'auteur, collectionneur hambourgeois dont le livre reproduit quelques objets ( 72 p. 149, 131 p. 215, 158 p. 248), s'y appuie sur les avancées récentes de la recherche germanophone et divers documents d'archives en sa possession. Si son travail donne un assez bon aperçu du «collecting colonialism» allemand, pour reprendre le titre du livre pionnier de Chris Gosden et Chantal Knowles (2001), sa lecture demande quelque patience, $\mathrm{du}$ fait de nombreuses inadvertances de composition ou de traduction.

«L'île Mioko» (p.16) est d'abord située «à l'extrémité sud de la Nouvelle-Irlande » puis, six lignes plus loin, comme il est exact, « dans les îles du Duc d'York », c'est-à-dire moins loin de la Nouvelle-Bretagne, et il en va souvent de même pour les personnages mis en scène. Ainsi des "méthodistes Wesleyan australiens " (sic) qui "arrivent en 1875 » dans ce même archipel sous la houlette de George Brown, resté toujours fier de sa naissance en Angleterre (1908, p. 3). Ainsi du juge puis gouverneur Hahl, d'abord assimilé aux collecteurs et marchands Hellwig et Thiel (p.22) et rétabli dans ses fonctions officielles deux pages plus loin. Il est abondamment question de la firme Godeffroy et de ses collecteurs (pp.11-13, etc.) mais c'est seulement à la fin du texte qu'est cité (p. 39) "un marchand du nom d'Umlauff à Hambourg ", comme si son rôle n'avait pas été d'importance comparable. Kl.-J. Krüger rappelle à juste titre que l'introduction en 1904 de la capitation (head-tax, Kopfsteuer, voir Hahl, 1980, p. 112) a accéléré le trafic d'objets d'art dans l'ensemble de la colonie allemande, mais il n'éclaire guère la nature de cette taxe en la dénommant «impôt électoral» (p. 38). À côté de 
précisions exactes et souvent intéressantes, l'auteur n'évite pas les affirmations gratuites, assurant par exemple que parmi les colons collecteurs d'objets, «seul un pour cent d'entre eux avaient un intérêt réel pour l'ethnographie» (p. 22). Ce chapitre étant dépourvu d'annotations et de sources détaillées (tout comme les photographies anciennes qui l'illustrent), son utilité s'en trouve malheureusement amoindrie.

7 Lui fait suite une évocation photographique de l'archipel et de ses habitants actuels (Bart Van Bussel, « Mes voyages en Océanie », pp. 42-49), autour de deux festivals, l'un à Tatau (l'une des îles Tabar, que ne situent ni le texte ni la carte ancienne reproduite en tête d'ouvrage) et l'autre en pays baining. Dans « L'art de l'archipel Bismarck et l'avantgarde » (pp. 50-65), K. Conru revient sur un pan bien connu de l'histoire des idées et de la sensibilité occidentales, la passion pour « les fétiches d'Océanie et de Guinée » que développèrent, après Apollinaire, beaucoup de grands artistes $\mathrm{du} \mathrm{xx}^{\mathrm{e}}$ siècle. Lire, dans le premier paragraphe (p.51), «Dürer, contemporain de Gilsemans » fait craindre le pire quand on se souvient que le premier est mort en 1528 et que le second, qui accompagna Tasman dans ses voyages, naquit seulement en 1606 (Anderson, 2001, p. 1). Heureusement, les développements consacrés à Emil Nolde, Roberto Matta, Serge Brignoni, Henry Moore et Anthony Caro sont plus solides, quoique forcément sujets à débat dans leurs appréciations et leurs interprétations esthétiques.

Le cœur du livre est constitué par les spectaculaires photographies en pleine page d'H. Dubois, ordonnées selon le même cheminement que le texte d'I. Heermann. Les objets, parfois reproduits plus grands que nature, sont généralement mieux visibles que dans les meilleurs musées, ce qui pourrait contribuer au succès de cette entreprise éditoriale. Conçues et retouchées à l'identique, dans un style où $\mathrm{H}$. Dubois excelle depuis longtemps - arrière-plans gris ou noirs, qu'anime de temps en temps un halo venu d'on ne sait où - ces images dessinent des liens factices entre des objets sans autres rapports entre eux que de s'être retrouvés réunis dans des vitrines de musées ou de collectionneurs les exposant selon les mêmes normes de présentation, d'éclairage, de température et d'hygrométrie, comme on voit au musée du quai Branly et ailleurs. Était-ce ainsi que leurs créateurs et utilisateurs les regardaient et les appréciaient? Si ce contexte échappe à la reconstitution, ces photographies certainement destinées à en exalter les formes et les couleurs finissent paradoxalement par les faire ressembler à des soldats à la revue que ne distinguent plus que des décorations variées sur leurs uniformes. Ces images sont bien sûr excellentes dans leur genre.

9 De haute venue et abondamment annoté quant à lui, le long texte d'I. Heermann («Traditions artistiques de l'archipel Bismarck», pp.66-317) ne manquera pas d'intéresser amateurs et spécialistes. Sa première section, «Découverte de la région » montre ce qui en fait la cohérence au-delà des quelques décennies de présence coloniale allemande lui ayant imposé ce nom. La section suivante, "Art et ethnographie ", s'attache au développement historique des connaissances occidentales sur les arts et les sociétés de l'archipel pour en souligner les tâtonnements et les limites qui lui font conclure, dans un paragraphe "plein d'usage et raison", par un de ces conditionnels qu'on nomme « potentiel»:

« La complexité des formes et des variantes, et les principes directeurs employés

dans la création d'œuvres d'art pourraient nous aider à comprendre ce qui est en

jeu dans ces sociétés et ces cultures prétendument plus simples.» (p. 83)

10 Suivent des exposés consacrés aux îles de l'Amirauté, aux îles occidentales et SaintMatthias, à la Nouvelle-Bretagne et enfin à la Nouvelle-Irlande. Selon le principe de ce 
type d'ouvrage, le texte mêle connaissances générales, citations de documents significatifs et commentaires des objets reproduits, de sorte que sa lecture s'apparente à une visite guidée, menée par l'une des meilleures spécialistes et analystes de ces arts. Le plus remarquable est qu'elle anime ces exposés documentaires en évoquant les hypothèses les plus sérieuses dont ces traditions artistiques et rituelles ont fait l'objet. Pour ne citer qu'un seul exemple, ses pages consacrées aux îles de l'Amirauté rappellent les convergences qu'elle avait déjà signalées avec les traditions micronésiennes (Heermann, 2002) mais aussi les conjectures de Christian Kaufmann sur un ancien fond austronésien expliquant l'attachement aux aménagements et aux constructions en hauteur du type plateforme (2002:49-50). Toute cette partie du livre un peu plus des trois quarts de la pagination - est parfaitement réussie. Elle suffit à justifier l'édition de ce coffee table book de luxe, dont il faut espérer que le format et le poids ne décourageront pas les lecteurs de se plonger dans l'excellent texte d'I. Heermann.

\section{BIBLIOGRAPHIE}

ANDERSON Grahame, 2001. The Merchant of the Zeehaen. Isaac Gilsemans and the voyages of Abel Tasman, Wellington, Te Papa Press.

BROWN George, 1908. Pioneer-Missionary and Explorer. An Autobiography, London, Hodder and Stoughton.

CONRU Kevin et Deborah WAITE, 2008. Trésors des îles Salomon. La collection Conru, photographies de Hughes Dubois, Milan, Éditions 5 Continents.

GOSDEN Chris and Chantal KNOWLES, 2001. Collecting Colonialism. Material Culture and Colonial Change, Oxford, Berg.

GUIART Jean, 1965. Nouvelles-Hébrides, Auvers-sur-Oise, Archée éditeur.

HAHL Albert, 1980. Governor in New Guinea, edited and translated by Peter G. Sack and Dymphna Clark, Canberra, ANU Press.

HEERMANN Ingrid (hrsg), 2001. Form Farbe Phantasie. Südsee-Kunst aus Neubritannien, Stuttgart, Linden-Museum/Arnoldsche.

-, 2002. Seen in a Wider Perspective : the art forms of the Admiralty Islands, in Christian Kaufmann, Christin Kocher-Schmid and Sylvia Ohnemus, Admiralty Islands. Art from the South Seas, Zurich, Rietberg Museum, pp. 92-98.

- (hrsg), 2009. Südsee-Oasen, Leben und Überleben im Westpazifik, Stuttgart, Linden-Museum. HERREMAN Frank (éd.), 2008. Océanie. Signes de rites, symboles d'autorité, Bruxelles, ING-Fonds Mercator.

KAUFMANN Christian, 2002. Arts of the Admiralty Islands : long overlooked, and more complex than most observers believe, in Christian Kaufmann, Christin Kocher-Schmid and Sylvia Ohnemus, Admiralty Islands. Art from the South Seas, Zurich, Rietberg Museum, pp. 42-52. 
MEYER Adolf Bernhard, 1889. Masken von Neuguinea und dem Bismarck-Archipel, Königliches Ethnographisches Museum zu Dresden, vii.

MEYER Adolf Bernhard und Richard Parkinson, 1895. Schnitzereien und Masken vom BismarckArchipel und Neu-Guinea, Publicationen aus dem Königlichen Ethnographischen Museum zu Dresden, $\mathrm{x}$.

PORTIER André et François Poncetton, 1930. Les Arts sauvages. Océanie, Paris, éditions Albert

Morancé.

—, 1931. Décoration océanienne, Paris, A. Calavas.

schmitz Carl A., 1962. Ozeanische Kunst. Skulpturen aus Melanesien, « Akanthus-Buchreihe zur Geschichte der Skulptur ", München, Verlag F. Bruckmann.

\section{NOTES}

1. Légendes et attributions dans l'ensemble correctes, quoique l'une prétende faire passer le Pirée pour un homme en affirmant d'une coupe sculptée dans la province de Manus, chef-lieu Lorengau, qu'elle a été « collectée par l'officier colonial Lorengau », avec « étiquette de la main de Lorengau, ms. $898 »(33$, p. 106 et 107). 